\title{
Algorithmic Meta Theorems
}

\author{
Martin Grohe \\ Institut für Informatik, Humboldt-Univerität zu Berlin \\ Unter den Linden 6, 10099 Berlin, Germany \\ grohe@informatik.hu-berlin.de
}

\begin{abstract}
Algorithmic meta theorems are algorithmic results that apply to whole families of combinatorial problems, instead of just specific problems. These families are usually defined in terms of logic and graph theory. An archetypal algorithmic meta theorem is Courcelle's Theorem [1], which states that all graph properties definable in monadic second-order logic can be decided in linear time on graphs of bounded tree width. More recent examples of such meta theorems state that all first-order definable properties of planar graphs can be decided in linear time [2] and that all first-order definable optimisation problems on classes of graphs with excluded minors can be approximated in polynomial time to any given approximation ratio $[3]$.

In my talk, I gave an overview of algorithmic meta theorems and the main techniques used in their proofs. Reference 4] is a comprehensive survey of the material.
\end{abstract}

\section{References}

1. Courcelle, B.: Graph rewriting: An algebraic and logic approach. In: van Leeuwen, J. (ed.) Handbook of Theoretical Computer Science, vol. B, pp. 194-242. Elsevier Science Publishers, Amsterdam (1990)

2. Frick, M., Grohe, M.: Deciding first-order properties of locally tree-decomposable structures. Journal of the ACM 48, 1184-1206 (2001)

3. Dawar, A., Grohe, M., Kreutzer, S., Schweikardt, N.: Approximation schemes for first-order definable optimisation problems. In: Proceedings of the 21st IEEE Symposium on Logic in Computer Science, pp. 411-420 (2006)

4. Grohe, M.: Logic, graphs, and algorithms. In: Flum, J., Grädel, E., Wilke, T. (eds.) Logic and Automata - History and Perspectives. Texts in Logic and Games, vol. 2, pp. 357-422. Amsterdam University Press (2007) 